\title{
Radiofrequency Catheter Ablation in Patients with Atrial Fibrillation
}

\author{
Mauricio Scanavacca, Eduardo Sosa, André D'Ávila, Fernando Tondato, Francisco Darrieux, \\ Denise Hachul, Antonio Bahia, Paulo Cavalcanti, Flávio Oliveira
}

São Paulo, SP - Brazil

Recent reports have demonstrated that, in some patients, atrial fibrillation is triggered by ectopic foci originated from the pulmonary veins and that these foci can be effectively treated by radiofrequency pulses. In this report we confirm these findings in three patients with paroxistic atrial fibrillation refractory to antiarrhythmic drugs, while we caution against the occurrence of pulmonary veno-occlusive syndrome, observed in one of the patients after the second attempt to treat atrial fibrillation with RF ablation. We concluded that although RF pulses are effective to interrupt focal atrial fibrillation, multiple applications in the pulmonary veins can produce significant pulmonary vein stenosis. The significance of these findings remains to be determined after a longer follow-up.

Atrial fibrillation (AF) is one of the most frequently found cardiac arrhythmia in clinical practice. Morbidity is associated to higher heart rates and to supervening thromboembolic phenomena ${ }^{1,2}$. Antiarrhythmic drugs can successfully prevent clinical recurrences in about $50 \%$ of the patients, but since its use implies in an additional risk of proarrhythmia, a definitive ablative procedure would thus be highly desirable ${ }^{3,4}$

An effective surgical alternative to maintain sinus rhythm has been introduced by Cox et al ${ }^{5}$, however, due to technical limitations ${ }^{6-8}$, it has not been adequately reproduced in the electrophysiologic (EP) laboratory. Haissaguerre et $\mathrm{al}{ }^{9}$ have recently demonstrated that, in a few patients, AF is originated from ectopic foci situated in the majority of the cases near the pulmonary veins, and that a relatively simple focal ablative radiofrequency procedure can prevent its recurrence in about $60 \%$ of the patients.

Although these results have brought a great deal of enthusiasm due to opening of new perspectives, a larger number of patients with a longer follow-up must be investigated before definitive conclusions are drawn. The objective of this study is to report the initial results with

Instituto do Coração do Hospital das Clínicas - FMUSP

Mailing adress: Mauricio Scanavacca - Incor - Av. Dr. Enéas C. Aguiar, $44-$ 05403-000 - São Paulo, SP - Brazil this novel technique, as well as to caution against the risk of developing pulmonary veno-occlusive syndrome, observed in one patient submitted to radiofrequency catheter ablation.

Case 1 - The first patient was a 36 year-old male who had presented with occasional episodes of paroxistic AF for the past ten years, and daily recurrences for the past two years. He had failed to respond to quinidine associated to digoxine, amiodarone, sotalol and subsequently propafenone associated to atenolol. After clinical and echocardiographic evaluation, no systemic or structural heart disease was documented. Rest ECG was normal, but ambulatory Holter monitoring displayed several episodes of non-sustained AF following atrial premature beats and frequent episodes of atrial tachycardia.

In June, 1998, an EP study was undertaken under deep sedation with propofol, midazolam and fentanyl. Three catheters were inserted percutaneously into the right femoral artery. A decapolar catheter was positioned at the lateral wall of the right atrium. An octapolar catheter was inserted into the coronary sinus, and a quadripolar catheter with a $4 \mathrm{~mm}$ tip was inserted in the left atrium, through a transeptal approach (fig. 1). An intravenous bolus of heparin (5000 IU) was given, followed by a 1000 $\mathrm{UI} / \mathrm{hr}$ continuous infusion.

AF was recorded in left atrial electrograms, whereas right atrium electrograms were organized and regular (fig. 2). Three direct current shocks $(200 \mathrm{~J})$ were delivered in an attempt to restore sinus rhythm, but that was maintained for only a few seconds, with AF recurrence (fig. 3). With the catheter positioned at the right inferior pulmonary vein, we observed that AF initiated after an atrial premature beat occurring earlier at that site when compared to the right atrial and coronary sinus signals. However, it did not precede peripheral $\mathrm{P}$ wave. The catheter was subsequently manipulated towards the left superior pulmonary vein (LSPV) ostium and another direct current $200 \mathrm{~J}$ shock delivered. After AF recurred, it could be observed that the atrial beat initiating the tachycardia was about $100 \mathrm{msec}$ earlier at this site than the $\mathrm{P}$ wave preceding the tachycardia (fig. 4). A 

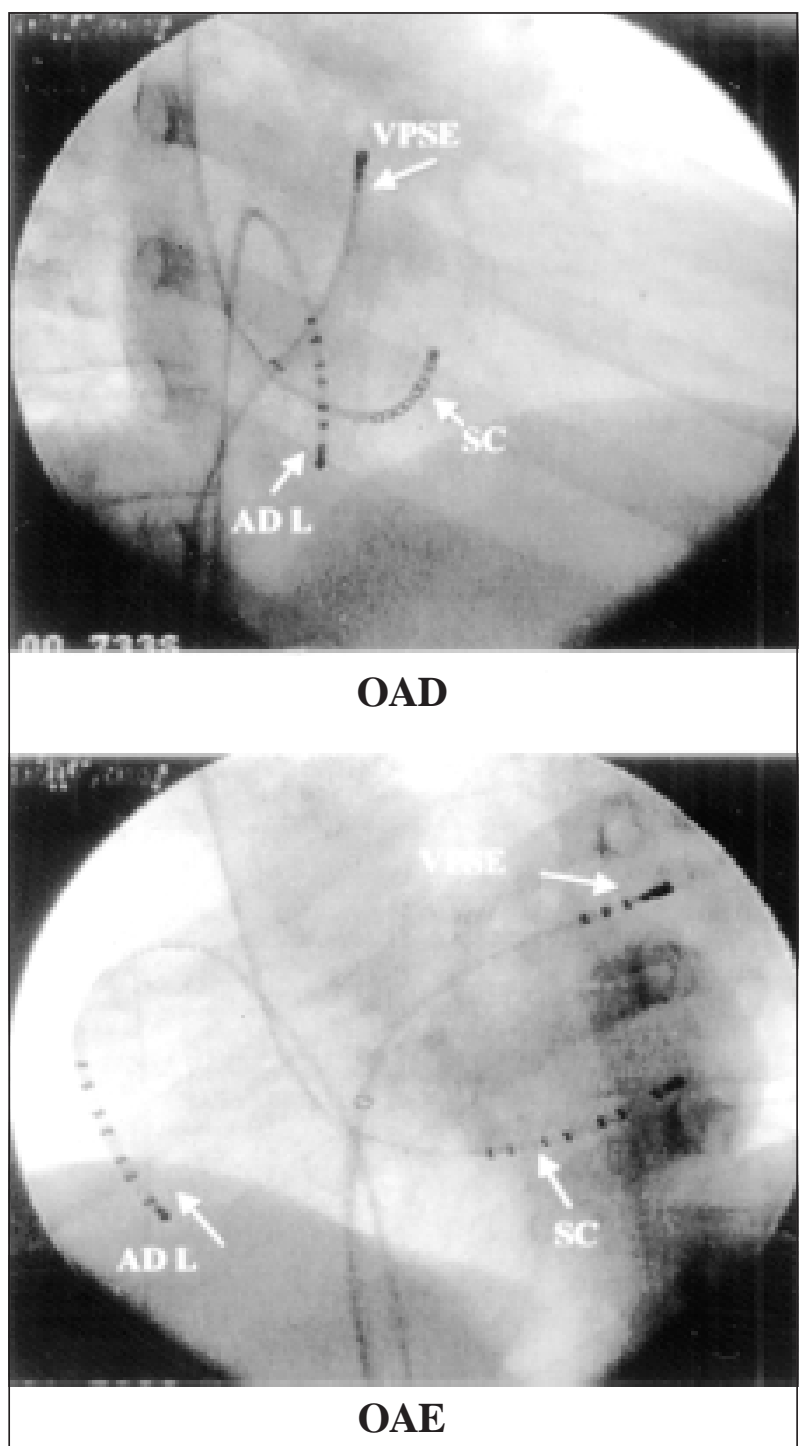

Fig. 1 - Fluoroscopic image of electrophysiologic catheters in right (RAO) and left (LAO) anterior oblique projections. ADL: octapolar catheter positioned on the right atrial lateral wall parallel to the tricuspid valve annulus. SC: octapolar catheter positioned in the coronary sinus. VPSE: quadripolar catheter with a $4 \mathrm{~mm}$ tip inserted in the left superior pulmonary vein ostium through a trans-septal approach.

radiofrequency $(\mathrm{RF})$ pulse of $60^{\circ} \mathrm{C}$ was delivered at that site immediately after spontaneous onset of AF. There was no modification of cardiac rhythm during the first 15 seconds; the catheter was therefore moved slightly into the left superior pulmonary vein and two seconds later, AF was interrupted (fig. 5). Control EP study did not show accessory pathways or dual AV node. AF could still be induced by rapid atrial stimulation, but it was non-sustained. Contrast media was injected into the pulmonary veins, showing discrete left superior pulmonary vein dilation. Twenty fours hours after the procedure the patient presented a new episode of AF following a rapid atrial tachycardia, as shown by Holter monitoring. The patient was discharged on propafenone and atenolol and salicilic acid (200mg/ day). He was free of symptoms for about 40 days, when daily episodes of AF recurred. He was admitted to hospital for a new procedure after antiarrhythmic drugs were descontinued.

A new procedure using a similar protocol was repeated in October, 1998. During catheter insertion and manipulation in the coronary sinus, the patient remained in AF, but sinus rhythm was restored after positioning the quadripolar catheter inside the LSPV ostium. Sinus rhythm was maintained after isoproterenol, adenosine and programmed atrial stimulation. A venogram performed during the study did not show any LSPV modifications. Several RF pulses were delivered at that site (26 applications, ranging from 54 to 64 degrees Celsius, during 17 to 60 seconds) in order to isolate the LSPV ostium. The reason for these applications was that an electric potential was detected inside the pulmonary vein and there was atrial capture after stimulation of this site. The patient was discharged on a regimen of atenolol $(50 \mathrm{mg} /$ daily) and aspirin (200mg/daily), but was readmitted to hospital ten days later due to chest pain and hemoptysis. Physical examination, ECG and plain chest radiography revealed normal findings. Radionuclide ventilation-perfusion lung scan demonstrated a normal pattern of ventilation in both lungs, with diminished perfusion to the left lung. Ultrasound of lower extremities (at the site of venopuncture) disclosed no abnormalities. Fiberoptic bronchoscopic examination showed bleeding within the bronchus related to the lingula. Spiral (helical) computer tomography showed normal arterial flow to the lungs with no evidence of pulmonary thromboembolism, but an opacity in the area of the inferior left lobe related to the lingula. Finally, transesophageal echocardiography (TEE) showed severe stenosis of the LSPV, and an image suggestive of thrombus close to its ostium. Systemic anticoagulation was initiated with intravenous heparin, with symptoms disappearing after one week anticoagulation. A balloon angioplasty of both left pulmonary veins was thus successfully performed with disappearance of the abnormal gradient. TEE, repeated 7 days after angioplasty, did not detect any LSPV gradient. Ambulatory Holter analysis showed frequent atrial premature beats but no episode of AF or atrial tachycardia. The patient is now asymptomatic and is being administered oral anticoagulants but no antiarrhythmic drug therapy.

Case 2 - A 62 year-old female with mild hypertension, had a 10 year-history of AF episodes, characterized by rapid self-limited palpitations, lasting from a few hours to 3 to 4 days. Electrical cardioversion (ECV) had been unsuccessfully attempted. Eventually, the arrhythmia spontaneously reverted to sinus rhythm during follow-up. She initially used beta-blockers, but due to frequent recurrences, amiodarone was administered (200 to 400mg/daily). However, she still had AF episodes once or twice a week. ECG displayed sinus rhythm and an intra-atrial conduction disturbance. Echocardiogram was normal, without left ventricular hypertrophy or left atrial enlargement. Ambulatory Holter recor- 


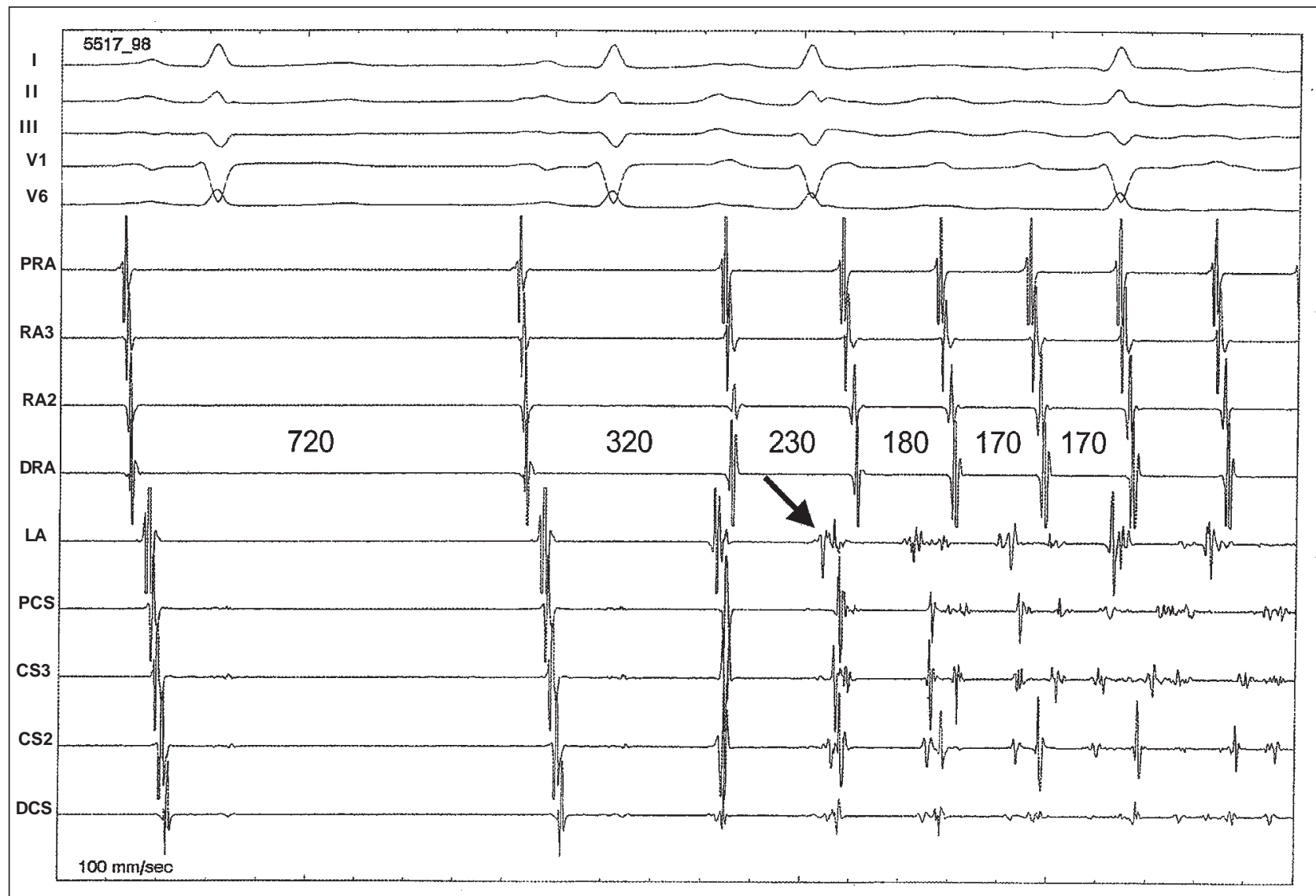

Fig. 2 - Atrial activation sequence from the onset of atrial fibrillation during EP study. I, II, III and V1: body surface electrocardiographic leads. PRA, RA3, RA2 and DRA: octapolar catheter electrograms from the right atrial lateral wall, from proximal (PRA) to distal (DRA) right atrium. PCS, CS3, CS2 and DCS: coronary sinus electrogram recorded with the octapolar catheter, showing the activation sequence from proximal (PCS) to distal (DCS) coronary sinus. LA: left atrialelectrograms from LSPV ostium (arrow). Note that the premature atrial beat initiating atrial fibrillation occurs earlier at the pulmonary vein ostium and that successive firing from a pulmonary vein focus produces a disorganized rhythm (atrial fibrillation) in the left atrium, whereas the right atrium is organized and regular. Paper speed: $100 \mathrm{~mm} / \mathrm{sec}$. Intervals are expressed in msec.

dings showed sinus rhythm with frequent premature atrial beats (PAB) and isolated episodes of non-sustained atrial tachycardia. In September, 1998, she was submitted to an EP study, during which only AF could be induced. She presented frequent monomorphic atrial premature beats, however. The earliest APB was situated at the LSPV ostium, where a $60^{\circ} \mathrm{C}$ RF pulse was delivered. A rapid atrial tachycardia started at the onset of the RF pulse, but it ceased following maintenance of the RF pulse application (fig. 6). After a 60 second RF pulse application, sinus rhythm was maintained and PAB disappeared. The patient was asymptomatic, without anti-arrhythmic drugs, when clinically evaluated 30 and 90 days after the procedure.

Case 3 - The third patient, a 36 year male, had been presenting with rapid palpitations lasting 1 to 48 hours (mostly 4 to 8 hours), for 16 years. He did not correlate them with any predisposing factors, and the arrhythmia reverted spontaneously after resting (he did not seek for medical help). AF episodes were documented when they lasted more than 24 hours and the patient would seek medical help for chemical cardioversion. At that point, he had tried several drugs, including digoxine associated to quinidine, beta-blockers and amiodarone. He had also been administered sotalol and propafenone for a short time, without success. ECG and echocardiogram disclosed no abnormalities. Ambulatory Holter monitoring showed frequent episodes of PAB and non-sustained AF. Laboratory exams such as thyroid hormones revealed no abnormalities. In 1998, the patient was submitted to a RF ablation in an attempt to ablate AF of possible focal origin. During the EP study the patient was in AF, since it had started the previous night. A $200 \mathrm{~J}$ electrical cardioversion was performed, and the explorer catheter maintained in the LSPV ostium. Sinus rhythm persisted for only a few seconds, though. AF recurred, induced by PAB occurring earlier in the LSPV than in the right atrium or coronary sinus. The catheter was positioned in the four pulmonary veins ostia and ECV repeated. The earlier site obtained was at the left inferior pulmonary vein (LIPV) ostium. Accordingly, two initial RF pulses $(60 \mathrm{~J})$ were delivered at that site for 10 seconds, without AF interruption. After a slight catheter manipulation in the 


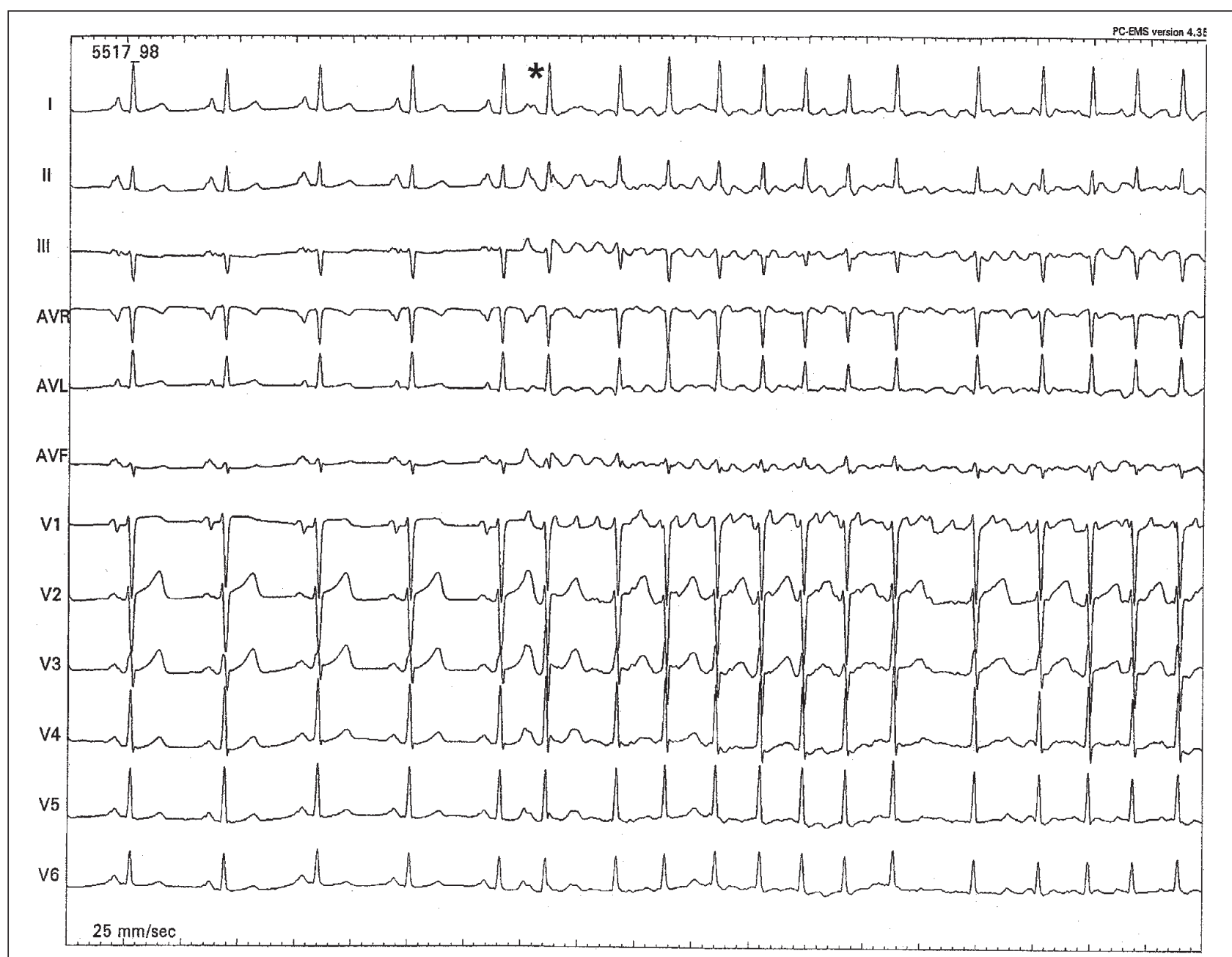

Fig. 3 - Spontaneous onset of atrial fibrillation after electric cardioversion. Note that atrial fibrillation is initiated after a premature atrial beat (*). Simultaneous recording of twelvelead electrocardiogram. Paper speed: $25 \mathrm{~mm} / \mathrm{sec}$.

same region, a third RF pulse, prolonged for 60 seconds, could interrupted AF in 5 seconds. Sinus rhythm was maintained and programmed electrical stimulation subsequently performed did not induce any tachycardia except for a type I atrial flutter, which was interrupted after a rapid atrial stimulation. Twenty four hours after the procedure the patient had a new episode of AF lasting for two hours, and recurring the following days. On the fourth day after the EP study, a new procedure was performed. A RF application pulse was delivered at the same site (LIPV), without AF interruption $\left(30 \mathrm{sec}, 60^{\circ} \mathrm{C}\right.$ ). The catheter was repositioned into the four pulmonary veins and ECV repeated. As before, the earliest atrial premature beat initiating AF was presumably situated into the LIPV ostium. AF was interrupted after a RF application pulse delivered in between the LSPV and LIPV ostia. Four more applications lasting for 60 seconds were delivered at that site. Sinus rhythm persisted during 48 hours, and the patient was discharged under a regimen of propranolol (20mg bid) and aspirin, 100mg daily. No AF was documented during a three month follow-up. Ambu- latory Holter monitoring showed sinus rhythm and isolated atrial premature beats. The patient is being followed at the out-patient clinic.

\section{Discussion}

These reports confirm the hypothesis that the pathophysiology of AF can involve a focal mechanism ${ }^{10-14}$ originating inside the pulmonary veins ${ }^{15-18}$, which is amenable to RF catheter ablation. Furthermore, it cautions about the risk of pulmonary veins stenosis related to the procedure.

Although this focal mechanism of AF originating in the pulmonary veins has opened new perspectives for alternative therapies such as RF ablation, many questions have still to be answered before it can be currently performed in the EP laboratory. In this sense, we believe our preliminary findings justify reporting these three cases to present the technique used and short-term safety of the procedure as well as to help selecting adequate patients for it.

Criteria for the selection of patients included clinical features suggesting a focal mechanism for AF, that is, patients with no structural heart disease, who had paroxistic 


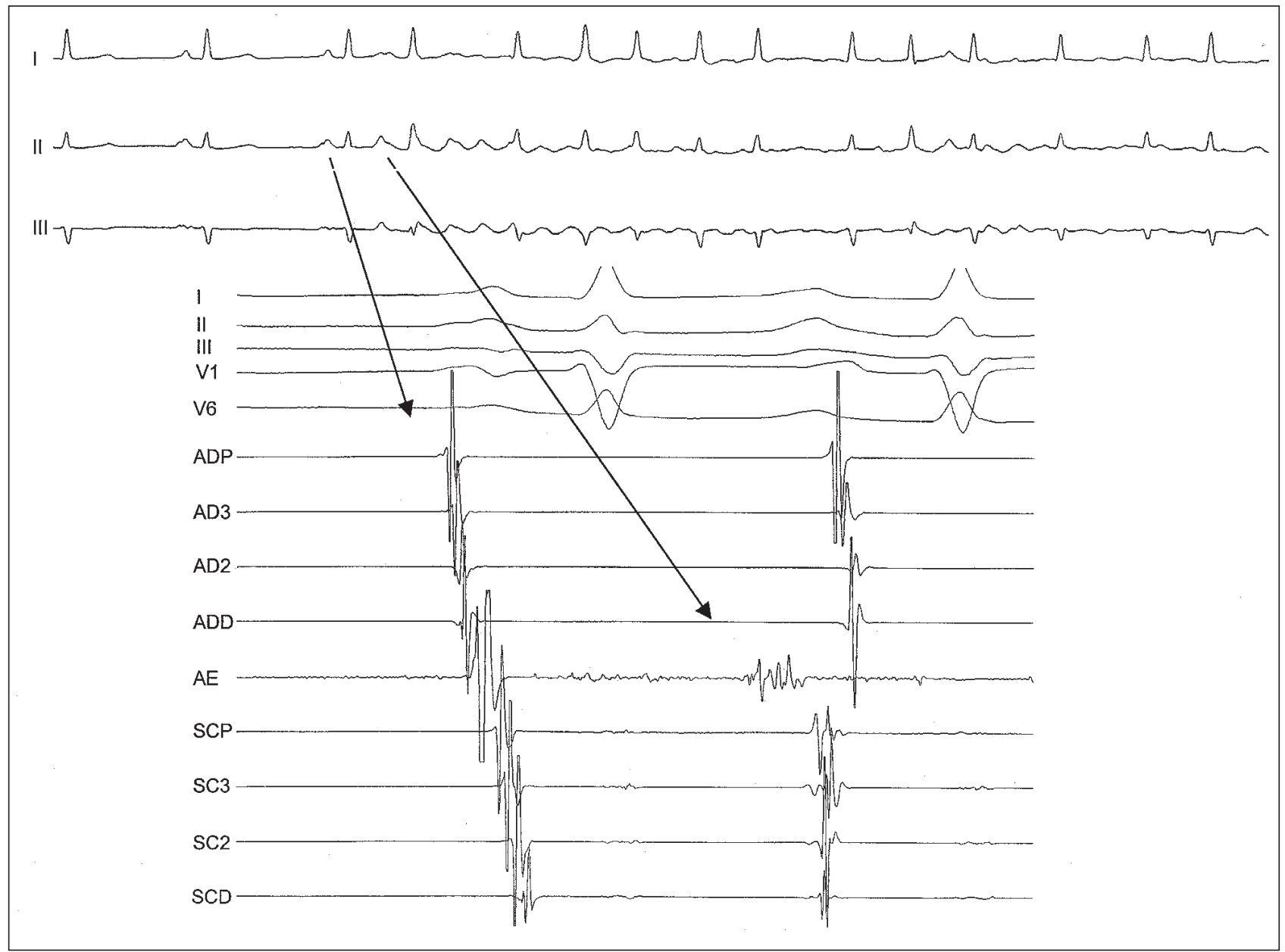

Fig. 4 - Electrogram at the site of RF application. Note that atrial premature beat that initiates atrial fibrillation is regis tered earlier when compared to the remaining electrograms. I, II, III, V1 and V6: body surface electrocardiographic leads; PRA, RA3, RA2, DRA: intracavitary right atrial electrograms, from proximal (PRA) to distal (DRA) right atrium. LA: left atrial electrograms (left upper pulmonary vein). PCS, CS3, CS2 and DCS: coronary sinus electrograms, showing the activation sequence from proximal (PCS) to distal (DCS) coronary sinus.

AF and an apparent active focal mechanism, such as frequent episodes of atrial tachycardia and atrial premature beats originating it. These criteria were effective for the three reported patients, since a focal mechanism was suggested in the EP study. However, these findings are rarely seen in patients with recurrent episodes of paroxistic AF. Arentz et al ${ }^{19}$ have shown atrial premature beats to occur in only $10 \%$ of 223 subjects with paroxistic AF. Therefore, although these features are highly specific, they very rarely occur, and most patients with paroxistic AF would not have an indication for RF ablation, according to the suggested criteria.

A very useful marker, in case it can be found, seems to be the site of spontaneous atrial premature beats or atrial tachycardia. In the second patient reported, the procedure was guided by the site where the earliest atrial premature beat could be mapped. An interesting finding was the appearance of a tachycardic rhythm induced by RF pulse and ceasing after it was maintained for some time. Its occurrence is similar to a phenomenon observed during RF application in tissues with intrinsic automaticity, such as the sinus node, the AV node and accessory nodes ${ }^{20}$. These observations suggest that atrial structures with automaticity could be involved in the mechanism of AF in some patients ${ }^{21}$.

Attempts to find a critical area for ablation in the first patient was an unusual experience, which was repeated in the third patient. Both of them could have a focal mechanism for AF clearly demonstrated. Our initial strategy was to map the origin of atrial premature beats and atrial tachycardia, fairly frequent in the first patient. Unfortunately, when the patient reached the EP laboratory, cardiac rhythm was sustained AF. Although we performed three ECV, sinus rhythm did not persist for a long time. On that occasion, we could see that AF was indeed interrupted by ECV, but a very fast rhythm originating close to the pulmonary veins reinduced the arrhythmia. We presumed that this rapid ectopic focus could be originating AF, and that it was probably maintained by a mechanism of reentry secondary to disorganized atrial depolarization. In order to be effective, RF application should interrupt AF from the start, so, as soon 


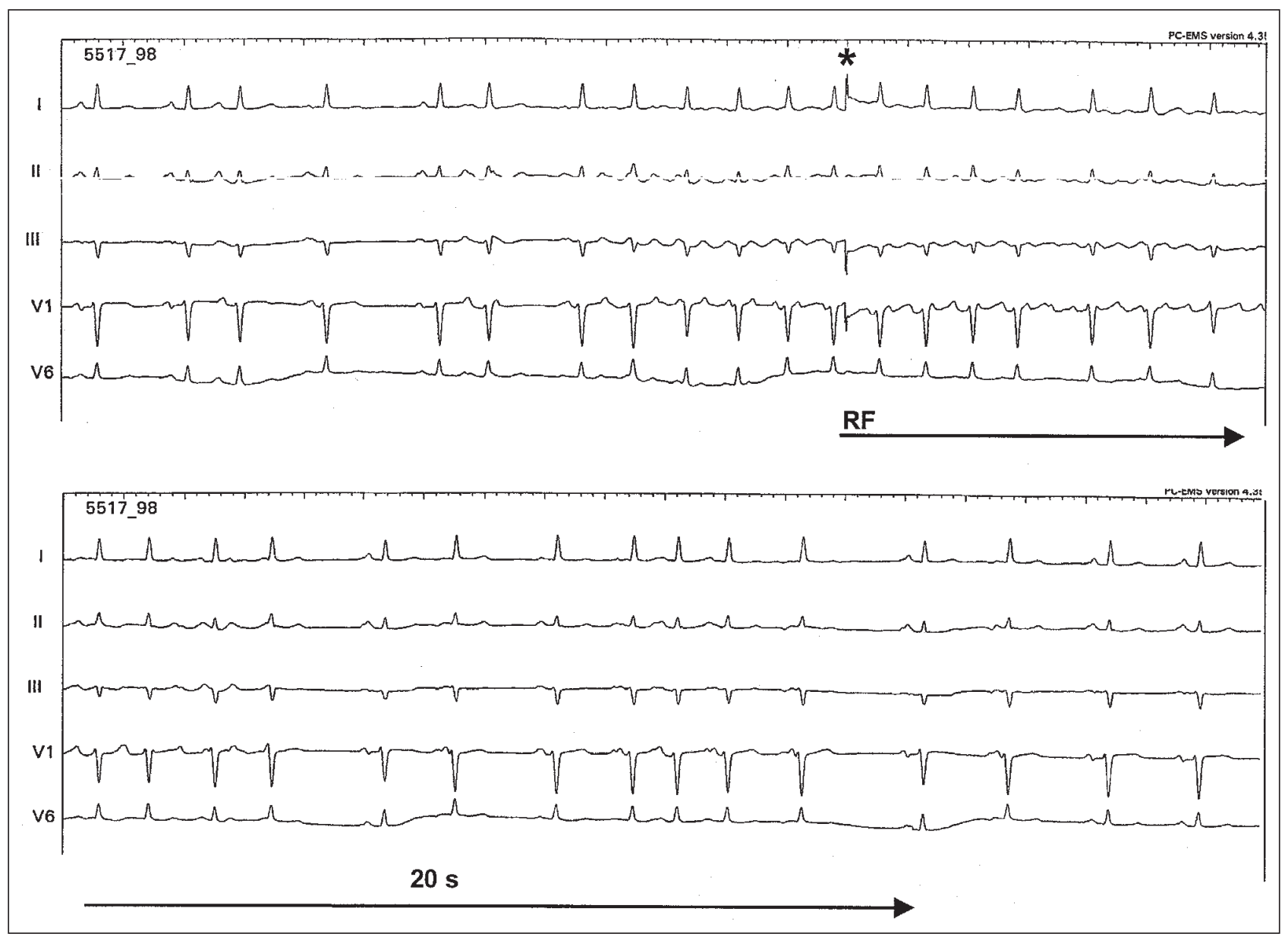

Fig. 5 - Atrial fibrillation interruption during RF pulse application. I, II, III, V1, and V6: body surface ECG leads, continuously recorded at the speed of 25 mm/sec. RF: onset of RF application; $20 \mathrm{sec}$ : time to reverse to a stable sinus rhythm. Paper speed of $25 \mathrm{~mm} / \mathrm{sec}$.

the probable site of origin was found, a RF pulse was delivered. During the initial 15 seconds of RF application, AF was not interrupted. We concluded that another mechanism other than the focus ( possibly reentry), could be sustaining AF, and that the initial focus might not be important any longer. Surprisingly, we noticed that after we positioned the catheter a few millimeters inside the LSPV, AF was interrupted and sinus rhythm maintained. From these findings, three assumptions could be made: first, the focus originating AF was also capable of maintaining it by a repetitive firing. This hypothesis is consistent with Satoh's and Zipe's observations ${ }^{22}$, who have suggested a mechanism of focal triggered activity leading to a polymorphic atrial tachycardia as well as AF. Secondly, it is possible that a focal mechanism can cause chronic AF, and therefore, some of these patients might be candidates for a focal ablation. Finally, mapping the restart of AF could be used as a mapping technique for patients with AF. Recently, Lao et al ${ }^{23}$ have analyzed the restart of AF after ECV to check whether it could be used to find the site of origin of this arrhythmia. They have observed AF recurrence in 5 of the 22 patients studied, permitting the detection of the site of origin in these patients. Two of these patients had paroxistic AF, whereas three had chronic AF. Furthermore, AF reinduction due to an active ectopic focus might be of practical importance, since that might explain why occasionally, this arrhythmia is not interrupted after ECV, only to be spontaneously interrupted later or interrupted with the use of antiarrhythmic drugs. In spite of our initial enthusiasm, two of the patients had AF recurrence the subsequent day. During the second procedure, the site of origin was suggested to be at the same site for both patients. In the first patient, the simple contact of the catheter with the LSPV interrupted AF rendering it non-inducible. We decided to deliver several RF applications around areas surrounding the LSPV ostium, which could have led to the complication described. In the third patient, the second EP study was similar to the first one, and a control EP study only induced sustained type I atrial flutter. We decided not to produce a conduction block at the caval-tricuspid isthmus, since this arrhythmia was not spontaneously documented and the clinical value of this intervention remains to be elucidated. A significant modification of the mechanism triggering AF is likely to have occurred, since all patients were severely symptomatic before the ablation and there no arrhythmia recurrence was documented after the procedure. Although no complica- 


\section{A}

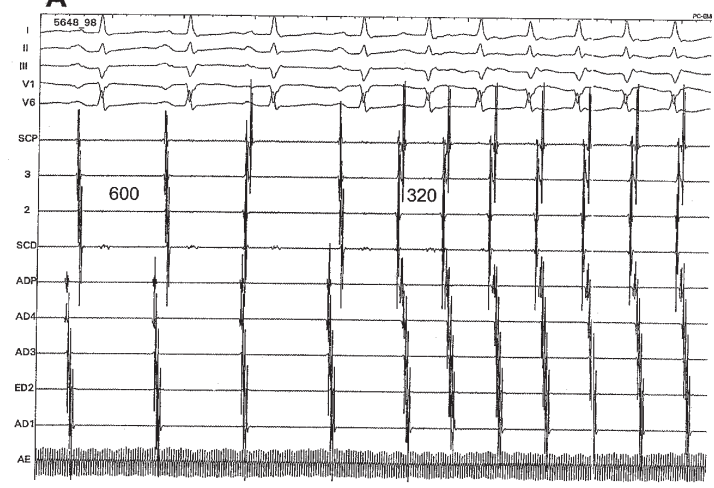

$50 \mathrm{~mm} / \mathrm{s}$

B

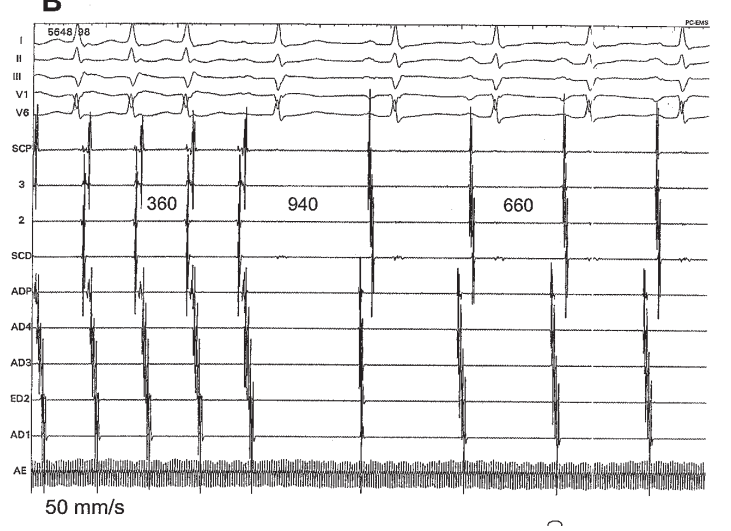

Fig. 6 - A) Atrial tachycardia occurring during RF application inside the left superior pulmonary vein ostium. I, II, III, V1, and V6: body surface ECG leads; PRA, RA3, RA2, DRA: intracavitary right atrial electrograms, from proximal (PRA) to distal (DRA) right atrium. LA: left atrial electrograms (left upper pulmonary vein). PCS, CS3, CS2 and DCS: coronary sinus electrograms, showing the activation sequence from proximal (PCS) to distal (DCS) coronary sinus. B: atrial tachycardia interruption occurring after continuous RF application. Paper speed of $50 \mathrm{~mm} / \mathrm{sec}$. Intervals are shown in msec. tions had been reported on previous studies, severe thrombosis and stenosis of LSPV ostium could be documented in one of our patients, leading to a pulmonary veno-occlusive vein syndrome with clinical repercussion, treated with balloon angioplasty. Although clinical symptoms could be acutely treated, long-term consequences are still to be defined. A recently published report ${ }^{24}$, has shown that our observations are not isolated. In this report, they attempted to isolate pulmonary veins in 18 patients, and in two patients severe pulmonary vein injury occurred after RF pulses. In both patients, there was extensive injury in all four pulmonary veins; clinical manifestations were more serious than those observed in our patient. Patients had to undergo catheterization with both dilation and stent positioning into the four veins.

In summary, we can assume a focal mechanism to be the cause of AF in patients without structural heart disease and paroxistic $\mathrm{AF}$ triggered by atrial ectopic beats and atrial tachycardia. The ectopic focus originating the arrhythmia can be detected by mapping the spontaneous site of origin of atrial premature beats or after mapping them when AF is interrupted. RF pulse application at the site of origin of these ectopic foci is capable of interrupting the arrhythmia and preventing its recurrence. However, multiple RF pulses in the pulmonary veins can produce severe stenosis and pulmonary veno-occlusive syndrome. The clinical importance of these observations can only be clearly defined after a long-term follow-up of these patients. Until these questions are fully answered, this procedure should be restricted to severely symptomatic patients refractory to drug therapy, selected and followed under a clinical investigation protocol.

\section{References}

1. Kannel WB, Abbott RD, Savage DD, McNamara PM. Epidemiologic features of atrial fibrillation. The Framingham Study. N Engl J Med 1982; 306: 1018-22.

2. Kopecky SL, Gersh BJ, McGoon MD, et al. The natural history of lone atrial fibrillation: a populational-based study over three decades. N Engl J Med 1987; 317: $669-74$

3. Jull-Möller S, Edvardsson N, Rehngvist-Alzlberg N. Sotalol versus quinidine for maintenance of sinus rhythm after direct current convertion of atrial fibrillation. Circulation 1990; 82: 1932-9.

4. Nguyen PT, Scheinman MM, Seger J. Polymorphous ventricular tachycardia: clinical characterization, therapy and the QT interval. Circulation 1986; 74: $340-9$.

5. Cox JL, Canavan TE, Shessler RB, et al. The surgical treatment of atrial fibrillation. II. Intraoperative mapping and description of the electrophysiologic basis of atrial flutter and fibrillation. J Thorac Cardiovasc Surg 1991; 101: 406-26.

6. Swartz JF, Pellersels G, Silvers J, et al. A catheter-based curative approach to atrial fibrillation in humans. Circulation 1994; 90(suppl I): I-335.

7. Haissagüerre M, Jais $P$, Shah DC, et al. Right and left atrial radiofrequency catheter therapy of paroxysmal atrial fibrillation. J Cardiovasc Electrophysiol 1996; 7 : 1132-44.

8. Kuck KH, Ernst S, Khanedani A, et al. Clinical Follow-up after Primary Catheter-Based Ablation of Atrial Fibrillation Using the CARTO System. Pacing Clin Electrophysiol 1998; 21: 868.

9. Haissaguerre M, Jais P, Shah DC, et al. Spontaneous initiation of atrial fibrillation by ectopic beats originating in the pulmonary veins. N Engl J Med 1998; 339: 659-66.
10. Haissagüerre M, Marcus FI, Fisher B, Clémenty J. Radiofrequency catheter ablation in unusual mechanisms of atrial fibrillation: report of three cases. $\mathrm{J}$ Cardiovasc Electrophysiol 1994; 5: 743-51.

11. Jais $P$, Haissagüerre $M$, Shah DC, et al. A focal source of atrial fibrillation treated by discrete radiofrequency ablation. Circulation 1997; 95: 572-6.

12. Sharma AD, Klein GJ, Guiraludon GM, Kiltein S. Atrial fibrillation in patients with Wolff-Parkinson-White syndrome: incidence after surgical ablation of the accessory pathway. Circulation 1985; 72: 161-9.

13. Chen PS, Pressley JC, Tang ASL, et al. New observations on atrial fibrillation before and after surgical treatment in patients with the Wolff-Parkinson-White syndrome. J Am Coll Cardiol 1992; 19: 974-8.

14. Konings KTS, Kirchhof CJHJ, Smeets , et al. High-density mapping of electrically induced atrial in humans. Circulation 1994; 89: 1665-80.

15. Nathan $\mathrm{H}$, Eliakin $\mathrm{M}$. The junction between the left atrium and the pulmonary veins. Circulation 1966; 34: 412-22.

16. Cavalcanti JS, Biazzoto W, Tavares DS, et al. Anatomia funcional da junção entre o átrio esquerdo e as veias pulmonares. Arq Bras Cardiol 1994; 62: 239-24.

17. Hatala R, Weiss C, Koschyk, et al. Radiofrequency catheter ablation of left atrial tachycardia originating within the pulmonary vein in a patient with dextrocardia. Pacing Clin Electrophysiol 1996; 6: 999-1002.

18. Alessie MA, Rensma PL, Brugada J, et al. Pathophisiology of atrial fibrillation. In: Zipes DP,Jalife J, eds - Cardiac Electrophysiology: From Cell to Bedside. Philadelphia: WB Saunders, 1990: 548-59. 
19. Arentz T, Ott P, Stockinger J, et al. Radiofrequency Catheter ablation of Focal Atrial Fibrillation. Pacing Clin Electrophysiol 1998; 21: 963.

20. Grogin HR, Lee RJ, Kwasman M, et al. Radiofrequency catheter ablation of atriofascicular and nodoventricular Mahaim tracts. Circulation, 1994; 90: 272-81.

21. Iwa T, Ichihashi T, Hashizume Y, et al. Successful surgical treatment of left atrial tachycardia. Am Heart J 1985; 109: 160-2.

22. Satoh T, Zipes DP. Cesium-induced atrial tachycardia degenerating into atrial fibrillation in dogs: atrial torsades de pointes? J Cardiovasc Electrophysiol 1998; 9: 970-5.

23. Lau CP, Tse HF, Ayers GM. Radiofrequency ablation of focal atrial fibrillation as guided by site of spontaneous reinitiation of atrial fibrillation after defibrillation. Pacing Clin Electrophysiol 1998; 21: 922.

24. Robbins IM, Colvin EV, Doyle TP, et al. Pulmonary vein stenosis after catheter ablation of atrial fibrillation. Circulation 1998; 98: 1769-75. 